Seventeen $(85 \%)$ of the subjects had a diagnosis of hypertension. Overall sleep quality was poor, with fragmentation and frequent arousals associated with respiratory events. Fifty-five percent of the patients had significant sleep-disordered breathing, mainly obstructive apnea. Both $\mathrm{BMI}$ and the prevalence of hypertension were similar in patients with and without sleep-disordered breathing.

The deceleration time, an index of diastolic dysfunction, was more prolonged in the group with sleep-disordered breathing $(236 \pm 40 \mathrm{~ms}$ vs $282 \pm 31 \mathrm{~ms} ; P<.05)$. As a group, a lower minimum percentage arterial oxygen saturation during sleep, but not AHI, was associated with more severe degree of diastolic dysfunction on echocardiogram, including a lower ratio between the early peak transmitted flow velocity and the late peak atrial systolic velocity $($ rho $=.57 ; P<.05)$ and a prolonged isovolumic relaxation time (rho $=-.54 ; P<.05$ ).

Sleep-disordered breathing is common in patients with DHF. In patients with DHF and sleep-disordered breathing, the associated diastolic dysfunction may be worse than in patients without sleep-disordered breathing. However, a causal relationship remains to be established.

Chan J, Sanderson J, Chan W, et al: Prevalence of sleep-disordered breathing in diastolic heart failure. Chest 1997;111:1488-1493.

\section{Zidovudine, didanosine, or both as initial treatment in symptomatic HIV-infected children}

Zidovudine has been the preferred drug for the initial treatment of symptomatic children infected with the human immunodeficiency virus (HIV). A multicenter, double-blind study was designed to assess the efficacy and safety of treatment with zidovudine alone or in combination with didanosine.

Children aged 3 months through 18 years were stratified according to age ( $<30$ months or $\geqslant 30$ months) and randomly assigned to receive zidovudine, didanosine, or zidovudine plus didanosine.
The primary end point was length of time to death or to progression of HIV disease.

A total of 831 children could be evaluated, of whom $92 \%$ had never received antiretroviral therapy and $90 \%$ had acquired HIV perinatally. An interim analysis (median follow-up, 23 months) showed a significantly higher risk of HIVdisease progression or death in the patients receiving zidovudine alone than in those receiving combination therapy (relative risk, $0.61 ; 95 \%$ confidence interval, 0.42 to $0.88 ; P=.007$ ).

The study arm with zidovudine alone was stopped and unblinded; the other two treatment arms were continued. At the end of the study, didanosine alone had an efficacy similar to that of zidovudine plus didanosine (median follow-up, 32 months) (relative risk of disease progression or death, $0.98 ; 95 \%$ confidence interval, 0.70 to $1.37 ; P=.91$ ). Patients receiving didanosine alone had a significantly lower risk of anemia or neutropenia $(P=.036)$.
Results of this study indicate that either didanosine alone or zidovudine plus didanosine was more effective than treatment with zidovudine alone in children with symptomatic HIV infection. The efficacy of didanosine alone was similar to that of the combined therapy and was associated with less hematologic toxicity.

Englund JA, Baker CJ, Raskino C, et al: Zidovudine, didanosine, or both as the initial treatment for symptomatic HIV-infected children. N EnglJ Med 1997;336:1704-1712.

\section{Orthostatic headaches and diffuse pachymeningeal gadolinium enhancement}

The authors describe their experience with 26 consecutive patients ( 15 men and 11 women aged 24 to 76 years) with orthostatic headaches and diffuse pachymeningeal gadolinium enhancement on magnetic resonance imaging (MRI). Clinical imaging and follow-up data were available for all these patients. Slide mate-

\section{DIRECTOR OF MEDICAL EDUCATION}

Northeast Regional Health System has an immediate opening for a Director of Medical Education. Northeast, the founding osteopathic institution, is located in Kirksville, Missouri, and is approximately 90 miles north of Columbia, Missouri. The area offers a variety of outdoor recreational activities and educational opportunities. Residents have access to larger cities while maintaining a small town quality of life.

This position reports to the Board of Directors and supervises Interns and the Department of Medical Education staff. The successful candidate must be a graduate from an AOA-approved medical school and have a minimum of 2 years experience. Salary is commensurate with experience.

Applicants should submit a letter of interest detailing experience and education, a complete curriculum vitae and three professional references to:

\author{
Northeast Regional Health System \\ P.O. Box C8502 \\ Kirksville, MO 63501 \\ Attn: Human Resources
}

\section{Northeast REgional}

$$
\text { HEALTHSYSTEM }
$$

